\author{
V. O. Yanchenko², Yu. A. Fedchenkova1', A. M. Demchenko ${ }^{1}$ \\ ${ }^{1}$ Nizhyn Mykola Gogol State University, Ukraine \\ 2, Grafska str., Nizhyn, 16600, Ukraine. E-mail: demch7758@ukr.net \\ ${ }^{2}$ T. H. Shevchenko National University "Chernihiv Colehium”, Ukraine
}

\title{
The synthesis and the study of the antitumor activity of 3-R-6-(4-methoxyphenyl)-7H-[1,2,4]triazolo[3,4-b][1,3,4]- thiadiazine hydrobromides
}

Aim. To synthesize and study the antitumor activity of 3-R-6-(4-methoxyphenyl)-7H-[1,2,4]triazolo[3,4- $b]$ $[1,3,4]$ thiadiazine derivatives.

Results and discussion. To determine the antitumor activity of 3-R-6-(4-methoxyphenyl)-7H-[1,2,4]triazolo[3,4-b][1,3,4]thiadiazine hydrobromides, the in vitro study was conducted on 60 lines of cancer cells (leukemia, non-small cell lung cancer, colon cancer, CNS cancer, melanoma, ovarian cancer, renal cancer, prostate cancer and breast cancer) according to the standard procedure of the mitotic activity assessment of new potential bioactive compounds by the fluorescent coloring method (sulforhodamine B as a dye). It was performed in the US National Cancer Institute within the Development Therapeutic Program. It has been found that derivatives of 3-R-6-(4-methoxyphenyl)-7H-[1,2,4]triazolo[3,4-b][1,3,4]thiadiazine exhibit the antineoplastic activity against a wide range of cancer cells lines and are promising core structures for creating new effective anticancer agents.

Experimental part. 3-R-6-(4-methoxyphenyl)-7H-[1,2,4]triazolo[3,4-b][1,3,4]thiadiazine hydrobromides were synthesized by the interaction of 4-amino-5-R-4H-1,2,4-triazole-3-thiols with 4-methoxyphenacyl bromide in ethyl acetate. The ${ }^{1} \mathrm{H}$ NMR spectra were recorded on a Bruker VXR-300 spectrometer (Germany) with the working frequency of $299.945 \mathrm{MHz}$.

Conclusions. A series of 3-R-6-(4-methoxyphenyl)-7H-[1,2,4]triazolo[3,4- $b][1,3,4]$ thiadiazine hydrobromides has been synthesized. The anticancer activity of the compounds obtained has been studied in the National Cancer Institute on 60 lines of tumor cells. Compounds that exhibit high levels of the antitumor activity have been found. It has been shown that the replacement of 3- $\mathrm{H}$ in compound $3 \mathrm{a}$ with ethyl or pentyl radicals leads to increase in the antitumor activity against MDA-MB-468 breast cancer cells.

Key words: $7 H$-[1,2,4]triazolo[3,4-b][1,3,4]thiadiazine; anastrozole; antitumor activity

В. О. Янченко른. Ю. Федченкова ${ }^{1}$, А. М. Демченко ${ }^{1}$

${ }^{1}$ Ніжинський державний університет імені Миколи Гоголя, Україна

${ }^{2}$ Національний університет «Чернігівський колегіум» імені Т. Г. Шевченка, Україна

Синтез та вивчення протипухлинної активності гідробромідів

3-R-6-(4-метоксифеніл)-7H-[1,2,4]триазоло[3,4-b][1,3,4]тіадіазину

Мета. Синтезувати та провести вивчення протипухлинної активності 3-R-6-(4-метоксифеніл)-7H-[1,2,4]триазоло[3,4-b][1,3,4]тіадіазинів.

Результати та їх обговорення. Для визначення протипухлинної активності гідробромідів 3-R-6-(4метоксифеніл)-7H-[1,2,4]триазоло[3,4-b][1,3,4]тіадіазинів проведено їx in vitro дослідження на 60 лініях ракових клітин (лейкемії, легень, товстого кишківника, ЦНС, меланоми, яєчників, нирок, простати, молочної залози) за стандартною процедурою оцінки мітотичної активності нових потенційних біологічно активних сполук методом фрлуоресцентного фрарбування (барвник - сульфородамін Б). Дослідження виконано в Національному інституті раку США (National Cancer Institute, USA) в рамках программи «Development Therapeutic Program». 3'ясовано, що похідні 3-R-6-(4-метоксифеніл)-7H-[1,2,4]триазоло[3,4-b][1,3,4]тіадіазину проявляють протипухлинну активність у широкому діапазоні ліній клітин раку і є перспективними базовими структурами для створення нових ефективних протипухлинних засобів.

Експериментальна частина. Гідроброміди 3-R-6-(4-метоксифеніл)-7H-[1,2,4]триазоло [3,4-b][1,3,4]тіадіазинів було синтезовано взаємодією 4-аміно-5-R-4H-1,2,4-триазол-3-тіолів з 4-метоксифенацилбромідом у середовищі етилацетату. ${ }^{1} \mathrm{H}$ ЯMP-спектри було зареєстровано на спектрометрі Bruker VXR-300 (Німеччина), робоча частота - 299,945 МГц.

Висновки. Синтезовано ряд гідробромідів 3-алкіл-6-(4-метоксифеніл)-7H-[1,2,4]триазоло[3,4-b][1,3,4]тіадіазинів. Вивчено протиракову активність одержаних сполук на 60 лініях пухлинних клітин у Національному інституті раку США. Ідентифіковано високоактивні сполуки, які проявили високий рівень протипухлинної активності. Доведено, що введення до базової сполуки етильного або пентильного радикалів у положення 3 гетероциклічної системи призводить до підвищення ії протипухлинної активності щодо клітин раку молочної залози MDA-MB-468.

Ключові слова: $7 H$-[1,2,4]триазоло[3,4-b][1,3,4]тіадіазин; анастрозол; протипухлинна активність 
Cancer is the second most important cause of mortality in the world. Thus, in 2018, 9.6 million people died from the disease according to the WHO. About one third of deaths from cancer are due to five major sources of risk - a high body mass index, low levels of fruit and vegetable consumption, the lack of physical activity, smoking and excessive alcohol use. Smoking is the most significant risk factor for cancer development, accounting for almost $22 \%$ of the world deaths from cancer [1]. Up to $25 \%$ of cases of cancer in low- and middle-income countries are due to infections, such as hepatitis and human papillomavirus [2]. The prostate and lungs in men and the mammary gland in women are the most commonly affected by cancer.

Nowadays, cyclophosphamide, methotrexate, vincristine, adriablastin are widely used to treat tumor diseases. These drugs exhibit the necessary healing properties, but have significant side effects on the hematopoietic system (leukopenia, anemia, thrombocytopenia), central nervous system (feeling tired, dizziness, headache, aphasia, drowsiness, seizures), reproductive system (disorder of oogenesis and spermatogenesis, oligospermia, menstrual irregularity, decreased libido, impotence), urinary system (hematuria, cystitis, severe renal dysfunction), allergic and dermatological reactions, etc. In this way, the search for new highly effective antitumor drugs remains a pressing issue today.

Previously, triazole derivatives have been proven to have the antitumor activity. The known drug anastrozole [3-7] (a derivative of 1,2,4-triazole) is active against estrogen dependent tumors of the breast in women. It is a selective non-steroidal enzyme antagonist of aromatase, which leads to a decrease in estradiol levels in peripheral tissues.

It is also known that derivatives of triazole and thiadiazine demonstrate a wide range of biological effects [8-10]. On the other hand, derivatives of $7 \mathrm{H}$ $[1,2,4]$ triazolo[3,4- $b][1,3,4]$ thiadiazine exhibit the antimicrobial, antifungal activity [11-12] and anti-inflammatory activity [13].

\section{Results and discussion}

Target $7 H$ - $[1,2,4]$ triazolo $[3,4-b][1,3,4]$ thiadiazine hydrobromides $3 \mathbf{a}-\mathbf{g}$ were synthesized by the interaction of 4-amino-5-R-4H-1,2,4-triazole-3-thiols 2a-g with 4-methoxyphenacyl bromide with relatively high yields (Scheme). Starting triazoles $\mathbf{2} \mathbf{b}-\mathbf{g}$ were obtained by the reaction of thiocarbohydrazide (1) with the corresponding carboxylic acids, for 5-unsubstituted triazole 2a formamide was used as a condensing reagent.

To determine the antineoplastic activity of 3-R-6(4-methoxyphenyl)-7 $H$-[1,2,4] triazolo[3,4- $b][1,3,4]$ thiadiazine hydrobromides 3 the in vitro study was conducted on 60 lines of cancer cells (leukemia, nonsmall cell lung cancer, colon cancer, CNS cancer, melanoma, ovarian cancer, renal cancer, prostate cancer and breast cancer) according to the standard procedure of the mitotic activity assessment of new potential bioactive compounds by the fluorescent coloring method (sulforhodamine B as a dye) performed in the US National Cancer Institute within the Development Therapeutic Program [14]. The substances were used in the concentration of $10^{-5} \mathrm{~mol} / \mathrm{L}$, cancer cells were incubated with the compounds for 48 hours. The results of the studies conducted were expressed as a percentage of the cancer cell growth compared to the reference drug 5 -fluorouracil (Table 1). In vitro experiments revealed high levels of the anti-tumor activity of the test compounds against almost all lines of cancer cells.

According to the Table 1 compounds $\mathbf{3 c}-\mathbf{g}$ possessed higher levels of the antineoplastic activity against cells of leukemia, non-small cell lung cancer, colon cancer, CNS cancer, melanoma, ovarian cancer, renal cancer, prostate cancer and breast cancer compared to those of the reference drug 5 -fluorouracil. Compounds $\mathbf{3 a}$,b showed the activity at the level of the reference drug. Compound $\mathbf{3 a}$ exceeded the activity of the reference drug for 23 lines of cancer cells, $\mathbf{3 b}$ for 21 lines, 3c - for 51 lines, 3d - for 52 lines, $\mathbf{3 e ~ - ~}$ for 59 lines, $\mathbf{3 f}$ - for 54 lines, and $\mathbf{3 g}$ - for 57 lines among 60 lines studied.

Compounds 3c-g inhibited MDA-MB-435 melanoma cells growth at the levels of $-13.02 \%,-0.35 \%$, $-7.41 \%,-2.71 \%$ and $-12.56 \%$, respectively, i.e. they not only stopped the growth and division of the cells, but also killed them.

It should be noted that compound 3c stopped the growth and division of MDA-MB-468 cells of breast cancer, and destroyed them at the level of $-0.91 \%$.

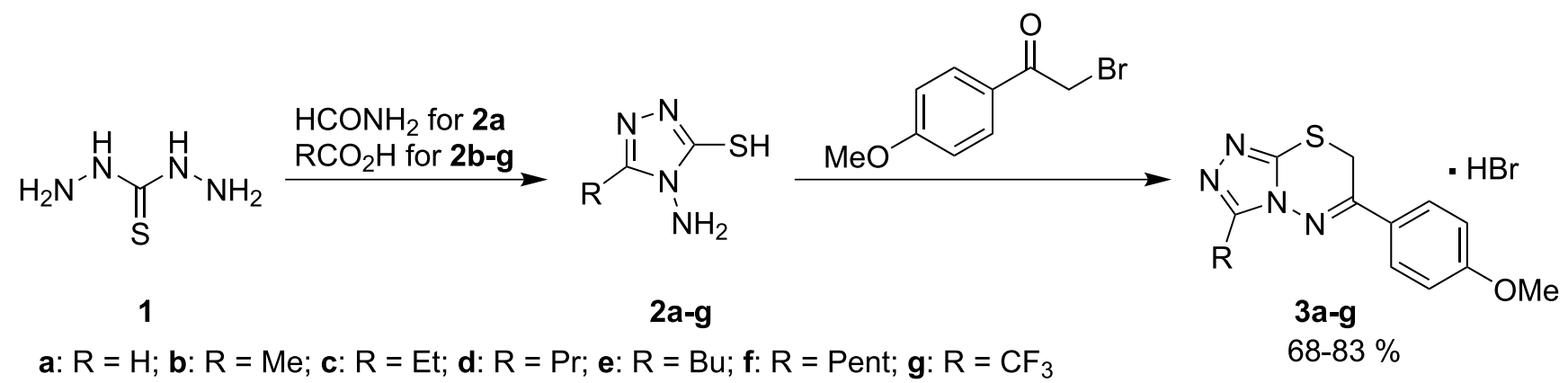

Scheme. The synthesis of 3-R-6-(4-methoxyphenyl)-7H-[1,2,4]triazolo[3,4-b][1,3,4]thiadiazine hydrobromides $\mathbf{3 a - g}$ 
Table 1

The anti-tumor activity of 3-alkyl-6-(4-methoxyphenyl)-7H-[1,2,4]triazolo[3,4-b][1,3,4]thiadiazine hydrobromides $\mathbf{3 a}-\mathbf{g}$

\begin{tabular}{|c|c|c|c|c|c|c|c|}
\hline \multirow{2}{*}{ The lines of cancer cells } & \multicolumn{7}{|c|}{ Compounds } \\
\hline & $3 a$ & $3 \mathbf{b}$ & $3 c$ & $3 d$ & $3 e$ & $3 f$ & $3 g$ \\
\hline 1 & 2 & 3 & 4 & 5 & 6 & 7 & 8 \\
\hline \multicolumn{8}{|c|}{ Leukemia } \\
\hline CCRF-CEM & 90.97 & 99.20 & - & 64.82 & 69.44 & 12.49 & 60.77 \\
\hline $\mathrm{HL}-60(\mathrm{~TB})$ & - & 85.83 & 46.93 & 55.20 & 27.90 & 7.13 & 60.75 \\
\hline K-562 & 109.18 & 108.09 & 20.53 & 16.06 & - & 12.75 & 16.29 \\
\hline MOLT-4 & 92.82 & 103.14 & 50.33 & 19.89 & 64.99 & - & 19.06 \\
\hline RPMI-8226 & 105.99 & 110.47 & 68.67 & 88.64 & 99.24 & 45.35 & 86.34 \\
\hline SR & 85.31 & 68.03 & 29.11 & 24.51 & 54.91 & 26.47 & 28.27 \\
\hline \multicolumn{8}{|c|}{ Non-Small Cell Lung Cancer } \\
\hline A549/ATCC & 94.22 & 90.34 & 49.88 & 68.67 & 67.22 & 30.09 & 60.62 \\
\hline EKVX & 87.61 & 92.21 & 78.15 & - & 74.90 & 45.54 & - \\
\hline HOP-62 & 97.65 & 95.18 & 42.83 & 61.26 & 73.46 & 34.62 & 66.67 \\
\hline HOP-92 & 109.07 & - & - & 64.29 & 98.87 & - & 76.47 \\
\hline $\mathrm{NCl}-\mathrm{H} 226$ & 95.69 & 97.84 & 78.24 & 77.45 & 83.71 & 67.42 & 90.26 \\
\hline $\mathrm{NCl}-\mathrm{H} 23$ & 106.43 & 110.63 & 71.33 & 64.42 & 81.91 & 44.23 & 76.58 \\
\hline $\mathrm{NCl}-\mathrm{H} 322 \mathrm{M}$ & 96.23 & 99.77 & 95.89 & - & 96.55 & 59.24 & 96.31 \\
\hline $\mathrm{NCl}-\mathrm{H} 460$ & 108.92 & 111.44 & 54.88 & 89.91 & 89.64 & 12.91 & 94.61 \\
\hline $\mathrm{NCl}-\mathrm{H} 522$ & 106.91 & - & & 59.15 & 85.24 & -0.85 & 52.83 \\
\hline \multicolumn{8}{|c|}{ Colon Cancer } \\
\hline COLO 205 & 117.74 & 111.53 & 49.96 & 61.02 & 91.43 & - & 81.03 \\
\hline HCC-2998 & 97.59 & 109.85 & 52.77 & 60.96 & 61.25 & 40.60 & 67.68 \\
\hline HCT-116 & 106.46 & 99.30 & 45.53 & 65.97 & 58.07 & 16.10 & 73.31 \\
\hline HCT-15 & 101.36 & 105.47 & 45.36 & 43.19 & 56.59 & 22.44 & 46.34 \\
\hline HT29 & 107.48 & 106.46 & 46.90 & 54.19 & 47.70 & 6.79 & 69.67 \\
\hline KM12 & 102.22 & 101.79 & 36.02 & 60.06 & 57.13 & 28.76 & 45.45 \\
\hline SW-620 & 107.50 & 108.91 & 43.64 & 40.15 & 32.79 & 18.25 & 38.02 \\
\hline \multicolumn{8}{|c|}{ CNS Cancer } \\
\hline SF-268 & 106.01 & 107.92 & 49.58 & 86.47 & 82.04 & 51.34 & 83.63 \\
\hline SF-295 & 100.52 & 115.19 & 64.89 & - & 76.33 & 4.79 & - \\
\hline SF-539 & 104.86 & 99.21 & 44.73 & 65.46 & 69.09 & 5.83 & 65.33 \\
\hline SNB-19 & 98.77 & 102.23 & 51.83 & - & 82.55 & 43.59 & - \\
\hline SNB-75 & 90.66 & 91.35 & - & 45.72 & 66.04 & 13.16 & 62.68 \\
\hline U251 & 95.74 & 95.61 & 54.54 & 68.94 & 64.69 & 38.41 & 58.31 \\
\hline \multicolumn{8}{|c|}{ Melanoma } \\
\hline LOX IMVI & 99.68 & 100.99 & 68.24 & 59.85 & 58.72 & 44.57 & 65.39 \\
\hline MALME-3M & 95.40 & 100.48 & 109.02 & - & 85.34 & 48.54 & 91.57 \\
\hline M14 & 103.00 & 99.31 & 46.09 & 55.11 & 43.34 & -1.10 & 58.63 \\
\hline MDA-MB-435 & 97.95 & 96.25 & -13.02 & -0.35 & -7.41 & -2.71 & -12.56 \\
\hline SK-MEL-2 & 128.42 & 137.89 & - & 62.81 & 98.22 & - & 63.37 \\
\hline SK-MEL-28 & 102.34 & 105.53 & 43.09 & 81.34 & 68.56 & 51.54 & 86.81 \\
\hline SK-MEL-5 & 101.46 & 102.54 & 27.35 & 72.16 & 80.99 & -0.14 & 80.05 \\
\hline UACC-257 & 103.06 & 102.85 & 67.04 & 98.52 & 86.31 & 71.97 & 83.63 \\
\hline UACC-62 & 103.32 & 102.85 & 50.51 & 73.46 & 73.59 & 46.77 & 67.24 \\
\hline
\end{tabular}


Continuation of Table 1

\begin{tabular}{|c|c|c|c|c|c|c|c|}
\hline 1 & 2 & 3 & 4 & 5 & 6 & 7 & 8 \\
\hline \multicolumn{8}{|c|}{ Ovarian Cancer } \\
\hline IGROV1 & 101.65 & 101.41 & 80.84 & - & 80.55 & 42.20 & 75.32 \\
\hline OVCAR-3 & 111.32 & 112.41 & 34.49 & 81.27 & 75.70 & -11.98 & 75.79 \\
\hline OVCAR-4 & 101.54 & 103.65 & 53.96 & 79.35 & 84.99 & 51.27 & 83.69 \\
\hline OVCAR-5 & 102.79 & 110.37 & 77.87 & 79.26 & 92.64 & 57.03 & 82.13 \\
\hline OVCAR-8 & 100.21 & 89.94 & 72.12 & 92.40 & 88.36 & 31.72 & 84.02 \\
\hline NCl/ADR-RES & 105.02 & 106.13 & 20.12 & 13.31 & 29.64 & 2.43 & 25.32 \\
\hline SK-OV-3 & 104.23 & 109.67 & - & 67.31 & 82.61 & - & 80.28 \\
\hline \multicolumn{8}{|c|}{ Renal Cancer } \\
\hline $786-0$ & 100.46 & 97.31 & 78.02 & 79.35 & 86.41 & 33.60 & 85.66 \\
\hline A498 & 87.40 & 101.18 & 48.97 & 37.81 & 49.40 & -1.24 & 10.40 \\
\hline $\mathrm{ACHN}$ & 109.65 & 100.21 & 58.58 & 81.69 & 85.73 & 45.24 & 84.80 \\
\hline CAKI-1 & 89.67 & 108.33 & 35.23 & 59.58 & 56.48 & 20.80 & 61.42 \\
\hline RFX 393 & 104.05 & 105.12 & 33.73 & 82.19 & 84.29 & 1.46 & 89.26 \\
\hline SN12C & 99.15 & 98.16 & 78.43 & 82.49 & 79.94 & 41.38 & 76.07 \\
\hline TK-10 & 103.75 & 109.96 & 38.63 & 75.90 & 90.87 & 29.99 & 80.05 \\
\hline UO-31 & 89.43 & 78.24 & 69.97 & - & 78.96 & 40.01 & 66.22 \\
\hline \multicolumn{8}{|c|}{ Prostate Cancer } \\
\hline PC-3 & 96.76 & 95.75 & 84.33 & 78.43 & 78.81 & 25.82 & 83.73 \\
\hline DU-145 & 114.20 & 114.68 & 74.30 & 110.63 & 92.29 & 16.17 & 94.00 \\
\hline \multicolumn{8}{|c|}{ Breast Cancer } \\
\hline MCF7 & 94.63 & 95.47 & 15.51 & 47.09 & 48.16 & 22.04 & 52.95 \\
\hline MDA-MB-231/ATCC & 109.69 & 103.56 & 55.33 & 76.52 & 71.09 & 44.68 & 74.19 \\
\hline HS 578T & 109.74 & 106.95 & 48.03 & 67.25 & 78.06 & 23.99 & 63.93 \\
\hline BT-549 & 96.41 & 103.10 & - & 69.29 & 68.19 & 26.08 & 73.02 \\
\hline $\mathrm{T}-47 \mathrm{D}$ & 99.26 & 89.91 & - & 67.02 & 90.75 & - & 78.22 \\
\hline MDA-MB-468 & 102.99 & 101.90 & -0.91 & 20.51 & 30.74 & -19.00 & 39.55 \\
\hline
\end{tabular}

Compound $3 f$ demonstrated the activity against NCI-H522 cells of non-small cell lung cancer at the level of $-0.85 \%$, SK-MEL-5 melanoma $-0.14 \%$, OVCAR-3 ovarian cancer $-(-11.98 \%)$, A498 renal cancer $-(-1.24 \%)$ and MDA-MB-468 breast cancer $-19.00 \%$.

Compound 3c was effective against K-562 and SR leukemia cells (exceeding the activity of 5-fluorouracil by 79.47 and $70.89 \%$, respectively), HOP-62 non-small cell lung cancer (exceeding 5-fluorouracil by $57.17 \%$ ), KM12 colon cancer (exceeding 5 -fluorouracil by $63.98 \%$ ), SF-26 and SF-539 CNS cancer (exceeding 5-fluorouracil by 50.42 and $55.27 \%$, respectively), SK-MEL-5 melanoma (exceeding 5-fluorouracil by $72.65 \%$ ), OVCAR-3 and NCI/ADR-RES ovarian cancer (exceeding 5 -fluorouracil by 65.51 and $79.88 \%$, respectively), CAKI-1, RFX 393 and TK-10 renal cancer (exceeding 5 -fluorouracil by 64.77, 66.27 and $61.03 \%$, respectively), MCF7 breast cancer (exceeding 5 -fluorouracil by $84.49 \%$ ).

Compound 3d was effective against K-562, MOLT-4 and SR leukemia cells (exceeding the activity of 5-fluorouracil by $83.94,90.11$ and $75.49 \%$, respectively),
HCT-15 and SW-620 colon cancer (exceeding 5-fluorouracil by 56.81 and $59.85 \%$, respectively), SNB-75 CNS cancer (exceeding 5 -fluorouracil by $54.28 \%$ ), NCI/ADR-RES ovarian cancer (exceeding 5-fluorouracil by $56.69 \%$ ), A498 renal cancer (exceeding 5 -fluorouracil by $62.19 \%$ ), MDA-MB-468 breast cancer (exceeding 5-fluorouracil by $79.49 \%$ ).

Compound 3e was effective against HL-60(TB) leukemia cells (exceeding the activity of 5 -fluorouracil by $72.10 \%$ ), SW-620 colon cancer (exceeding 5 -fluorouracil by $67.21 \%$ ), NCI/ADR-RES ovarian cancer (exceeding 5-fluorouracil by 70.36\%), MDA-MB-468 breast cancer (exceeding 5 -fluorouracil by 69.26\%).

Compound $\mathbf{3 f}$ was effective against HL-60(TB) leukemia cells (exceeding the activity of 5 -fluorouracil by $92.87 \%$ ), NCI-H460 non-small cell lung cancer (exceeding 5-fluorouracil by $87.09 \%$ ), HT29 colon cancer (exceeding 5 -fluorouracil by $93.21 \%$ ), SF-295 and SF-539 CNS cancer (exceeding 5-fluorouracil by 95.21 and $94.17 \%$, respectively), NCI/ADR-RES ovarian cancer (exceeding 5 -fluorouracil by $97.57 \%$ ), RFX 393 renal cancer (exceeding 5-fluorouracil by 
98.54\%), PC-3 and DU-145 prostate cancer (exceeding 5-fluorouracil by 74.18 and $83.83 \%$, respectively), MCF7 breast cancer (exceeding 5 -fluorouracil by $77.96 \%$ ).

Compound $3 \mathrm{~g}$ was effective against K-562 and MOLT-4 leukemia cells (exceeding the activity of 5-fluorouracil by 83.71 and $80.94 \%$, respectively), SW-620 colon cancer (exceeding 5 -fluorouracil by $61.98 \%$ ), NCI/ADR-RES ovarian cancer (exceeding 5-fluorouracil by $74.68 \%$ ), A498 renal cancer (exceeding 5 -fluorouracil by $89.60 \%$ ), MDA-MB- 468 breast cancer (exceeding 5 -fluorouracil by $60.45 \%$ ).

At the second stage of the study the most active compounds 3c, $\mathbf{3 f}$ and $\mathbf{3 g}$ were tested in five concentrations in 10 -fold dilution series $(100 \mu \mathrm{m}, 10 \mu \mathrm{m}$, $1 \mu \mathrm{m}, 0.1 \mu \mathrm{m}$ and $0.01 \mu \mathrm{m}$ ) on the lines of human can- cer cells listed above. As a result of the experiment, three dose-dependent parameters were calculated: the $\mathrm{GI}_{50}$ value (the growth inhibitory activity, effective inhibition level) corresponded to the concentration of the compound causing 50\% decrease in the net cell growth, the TGI value (cytostatic activity) - the concentration of the compound resulting in the total growth inhibition (TGI), and the $\mathrm{LC}_{50}$ value (cytotoxic activity) the concentration of the compound causing a net $50 \%$ loss of initial cells at the end of the incubation period. If logarithmic values of the parameters studied $\left(\operatorname{lgGI}_{50}\right.$, $\operatorname{lgTGI}$ and $\operatorname{lgL} \mathrm{C}_{50}$ ) were less than -4.00 , the compound was considered to be active [14-16].

According to the screening results (Table 2), the compounds demonstrated the considerable level of the anti-

Table 2

The results of the in-depth in vitro screening of compounds $\mathbf{3 c}, \mathbf{f}, \mathbf{g}$ in the concentration gradient of $10^{-4}-10^{-8} \mathrm{M}$

\begin{tabular}{|c|c|c|c|c|c|c|c|c|c|}
\hline \multirow{3}{*}{ The lines of cancer cells } & \multicolumn{9}{|c|}{ Compounds } \\
\hline & \multicolumn{3}{|c|}{$3 c$} & \multicolumn{3}{|c|}{$3 f$} & \multicolumn{3}{|c|}{$3 \mathbf{g}$} \\
\hline & $\operatorname{lgGl}_{50}$ & IgTGI & $\operatorname{lgLC} C_{50}$ & $\left.\operatorname{lgGl}\right|_{50}$ & lgTGI & $\operatorname{lgLC} C_{50}$ & $\operatorname{lgGl}_{50}$ & IgTGI & $\operatorname{lgLC} C_{50}$ \\
\hline 1 & 2 & 3 & 4 & 5 & 6 & 7 & 8 & 9 & 10 \\
\hline \multicolumn{10}{|c|}{ Leukemia } \\
\hline CCRF-CEM & -5.32 & -4.00 & -4.00 & -5.80 & -4.71 & -4.00 & -5.25 & -4.00 & -4.00 \\
\hline HL-60(TB) & -5.20 & -4.19 & -4.00 & -5.60 & -5.10 & -4.00 & -4.92 & -4.00 & -4.00 \\
\hline K-562 & -5.78 & -4.00 & -4.00 & -5.51 & -4.87 & -4.00 & -6.19 & -4.00 & -4.00 \\
\hline MOLT-4 & -5.22 & -4.00 & -4.00 & -5.56 & -4.89 & -4.00 & -5.42 & -4.00 & -4.00 \\
\hline RPMI-8226 & -4.94 & -4.31 & -4.00 & -5.70 & -4.73 & -4.00 & -4.81 & -4.00 & -4.00 \\
\hline SR & -5.39 & -4.00 & -4.00 & -5.52 & -4.99 & -4.00 & -6.22 & -4.00 & -4.00 \\
\hline \multicolumn{10}{|c|}{ Non-Small Cell Lung Cancer } \\
\hline A549/ATCC & -4.90 & -4.00 & -4.00 & -5.73 & -4.38 & -4.00 & -5.26 & -4.00 & -4.00 \\
\hline EKVX & -5.11 & -4.00 & -4.00 & -5.29 & -4.36 & -4.00 & - & - & -4.00 \\
\hline HOP-62 & -5.01 & -4.00 & -4.00 & -6.05 & -4.84 & -4.33 & -4.92 & -4.01 & -4.00 \\
\hline HOP-92 & -5.39 & -4.00 & -4.00 & -5.74 & -4.87 & -4.25 & -6.10 & -4.72 & -4.00 \\
\hline $\mathrm{NCl}-\mathrm{H} 226$ & -4.49 & -4.00 & -4.00 & -5.36 & -4.68 & -4.23 & -4.20 & -4.00 & -4.00 \\
\hline $\mathrm{NCl}-\mathrm{H} 23$ & -5.24 & -4.00 & -4.00 & -5.75 & -4.52 & -4.00 & -5.14 & -4.00 & -4.00 \\
\hline $\mathrm{NCl}-\mathrm{H} 322 \mathrm{M}$ & -4.34 & -4.00 & -4.00 & -5.12 & -4.03 & -4.00 & -4.65 & -4.00 & -4.00 \\
\hline $\mathrm{NCl}-\mathrm{H} 460$ & -5.14 & -4.00 & -4.00 & -5.58 & -4.93 & -4.37 & -4.97 & -4.00 & -4.00 \\
\hline $\mathrm{NCl}-\mathrm{H} 522$ & -5.37 & -4.22 & -4.00 & -5.84 & -5.08 & -4.31 & -5.31 & -4.32 & -4.00 \\
\hline \multicolumn{10}{|c|}{ Colon Cancer } \\
\hline COLO 205 & -5.01 & -4.00 & -4.00 & -5.89 & -5.14 & -4.36 & -4.92 & -4.00 & -4.00 \\
\hline HCC-2998 & -4.91 & -4.26 & -4.00 & -5.78 & -4.95 & -4.36 & -5.00 & -4.00 & -4.00 \\
\hline HCT-116 & -5.16 & -4.00 & -4.00 & -6.04 & -4.90 & -4.42 & -5.06 & -4.00 & -4.00 \\
\hline HCT-15 & -5.17 & -4.00 & -4.00 & -5.93 & -4.66 & -4.00 & -5.31 & -4.00 & -4.00 \\
\hline HT29 & -5.20 & -4.00 & -4.00 & -6.29 & -4.97 & -4.31 & -5.02 & -4.00 & -4.00 \\
\hline KM12 & -5.26 & -4.00 & -4.00 & -6.02 & -4.77 & -4.31 & -5.31 & -4.00 & -4.00 \\
\hline SW-620 & -5.17 & -4.00 & -4.00 & -6.26 & -4.42 & -4.00 & -5.34 & -4.00 & -4.00 \\
\hline \multicolumn{10}{|c|}{ CNS Cancer } \\
\hline SF-268 & -4.84 & -4.00 & -4.00 & -5.26 & -4.58 & -4.08 & -4.92 & -4.00 & -4.00 \\
\hline SF-295 & -5.32 & -4.00 & -4.00 & -6.07 & -4.98 & -4.43 & -5.64 & -4.84 & -4.00 \\
\hline
\end{tabular}


Continuation of Table 2

\begin{tabular}{|c|c|c|c|c|c|c|c|c|c|}
\hline 1 & 2 & 3 & 4 & 5 & 6 & 7 & 8 & 9 & 10 \\
\hline SF-539 & -5.32 & -4.00 & -4.00 & -5.83 & -5.25 & -4.57 & -5.36 & -4.59 & -4.00 \\
\hline SNB-19 & -4.87 & -4.00 & -4.00 & -5.65 & -4.62 & -4.06 & -4.85 & -4.00 & -4.00 \\
\hline SNB-75 & -5.33 & -4.00 & -4.00 & -5.74 & -5.09 & -4.41 & -5.37 & -4.15 & -4.00 \\
\hline U251 & -5.11 & -4.00 & -4.00 & -6.01 & -4.80 & -4.34 & -5.10 & -4.00 & -4.00 \\
\hline \multicolumn{10}{|c|}{ Melanoma } \\
\hline LOX IMVI & -5.19 & -4.00 & -4.00 & -5.81 & -4.74 & -4.28 & -5.19 & -4.00 & -4.00 \\
\hline MALME-3M & - & - & - & -4.93 & -4.52 & -4.10 & -4.00 & -4.00 & -4.00 \\
\hline M14 & -5.00 & -4.00 & -4.00 & -6.24 & -4.84 & -4.36 & -5.32 & -4.00 & -4.00 \\
\hline MDA-MB-435 & -6.02 & -5.37 & -4.00 & -6.64 & -6.09 & -4.47 & -6.44 & -5.77 & -5.00 \\
\hline SK-MEL-2 & -4.80 & -4.00 & -4.00 & -6.05 & -4.72 & -4.27 & -5.52 & -4.46 & -4.00 \\
\hline SK-MEL-28 & -4.73 & -4.00 & -4.00 & -5.29 & -4.66 & -4.27 & -4.80 & -4.00 & -4.00 \\
\hline SK-MEL-5 & -5.50 & -4.73 & -4.00 & -5.74 & -4.94 & -4.47 & -5.29 & -4.00 & -4.00 \\
\hline UACC-257 & -4.44 & -4.00 & -4.00 & -4.84 & -4.47 & -4.09 & -4.00 & -4.00 & -4.00 \\
\hline UACC-62 & -4.98 & -4.00 & -4.00 & -6.07 & -4.78 & -4.26 & -5.31 & -4.00 & -4.00 \\
\hline \multicolumn{10}{|c|}{ Ovarian Cancer } \\
\hline IGROV1 & -4.80 & -4.00 & -4.00 & -5.42 & -4.48 & -4.00 & -5.02 & -4.00 & -4.00 \\
\hline OVCAR-3 & -5.18 & -4.00 & -4.00 & -5.91 & -5.44 & -4.00 & -5.23 & -4.51 & -4.00 \\
\hline OVCAR-4 & -4.45 & -4.00 & -4.00 & -5.29 & -4.15 & -4.00 & -4.46 & -4.00 & -4.00 \\
\hline OVCAR-5 & -4.49 & -4.00 & -4.00 & - & - & - & -4.51 & -4.00 & -4.00 \\
\hline OVCAR-8 & -4.69 & -4.00 & -4.00 & -5.49 & -4.63 & -4.00 & -4.68 & -4.00 & -4.00 \\
\hline NCl/ADR-RES & -5.54 & -4.00 & -4.00 & -6.48 & -5.15 & -4.00 & -5.48 & -4.05 & -4.00 \\
\hline SK-OV-3 & -5.41 & -4.35 & -4.00 & -5.92 & -5.07 & -4.00 & -5.06 & -4.00 & -4.00 \\
\hline \multicolumn{10}{|c|}{ Renal Cancer } \\
\hline $786-0$ & -4.73 & -4.00 & -4.00 & -5.29 & -4.69 & -4.30 & -4.71 & -4.00 & -4.00 \\
\hline A498 & -5.21 & -4.00 & -4.00 & -6.38 & -5.22 & -4.46 & -5.71 & -4.28 & -4.00 \\
\hline $\mathrm{ACHN}$ & -4.78 & -4.00 & -4.00 & - & - & - & -4.81 & -4.00 & -4.00 \\
\hline CAKI-1 & -5.34 & -4.00 & -4.00 & -5.92 & -4.57 & -4.00 & -5.16 & -4.00 & -4.00 \\
\hline RFX 393 & -5.53 & -4.58 & -4.00 & -5.84 & -5.25 & -4.57 & -5.66 & -4.67 & -4.00 \\
\hline SN12C & -5.05 & -4.00 & -4.00 & -5.57 & -4.69 & -4.27 & -5.02 & -4.00 & -4.00 \\
\hline TK-10 & -4.68 & -4.00 & -4.00 & -5.40 & -4.49 & -4.00 & -4.81 & -4.00 & -4.00 \\
\hline UO-31 & -5.15 & -4.00 & -4.00 & -5.38 & -4.66 & -4.20 & -5.02 & -4.00 & -4.00 \\
\hline \multicolumn{10}{|c|}{ Prostate Cancer } \\
\hline PC-3 & -4.85 & -4.00 & -4.00 & -5.35 & -4.46 & -4.00 & -4.73 & -4.00 & -4.00 \\
\hline DU-145 & -4.77 & -4.00 & -4.00 & -5.39 & -4.63 & -4.00 & -4.91 & -4.00 & -4.00 \\
\hline \multicolumn{10}{|c|}{ Breast Cancer } \\
\hline MCF7 & -5.33 & -4.00 & -4.00 & -6.17 & -4.74 & -4.24 & -5.33 & -4.00 & -4.00 \\
\hline MDA-MB-231/ATCC & -4.89 & -4.00 & -4.00 & -6.13 & -4.82 & -4.27 & -4.99 & -4.00 & -4.00 \\
\hline HS 578T & -4.44 & -4.00 & -4.00 & -5.64 & -4.43 & -4.00 & -4.92 & -4.00 & -4.00 \\
\hline BT-549 & -4.71 & -4.00 & -4.00 & -5.22 & -4.66 & -4.31 & -5.07 & -4.00 & -4.00 \\
\hline $\mathrm{T}-47 \mathrm{D}$ & -5.28 & -4.00 & -4.00 & -5.57 & -4.54 & -4.00 & -5.05 & -4.00 & -4.00 \\
\hline MDA-MB-468 & -5.72 & -5.05 & -4.00 & -6.47 & -5.40 & -4.15 & -5.75 & -5.09 & -4.00 \\
\hline
\end{tabular}

carcinogenic activity. Compound $3 \mathbf{f}$ showed the significant level of the anticarcinogenic activity against HOP-62 non-small cell lung cancer $\left(\operatorname{lgGI}_{50}=-6.05\right.$; $\left.\lg \mathrm{TGI}=-4.84 ; \operatorname{lgLC}_{50}=-4.33\right)$, $\mathrm{HOP}-92(\operatorname{lgGI}=-5.74$; $\left.\lg \mathrm{TGI}=-4.87 ; \operatorname{lgLC}_{50}=-4.25\right), \mathrm{NCI}-\mathrm{H} 226\left(\operatorname{lgGI}_{50}=-5.36\right.$;
$\left.\operatorname{lgTGI}=-5.28 ; \operatorname{lgLC}_{50}=-4.27\right), \mathrm{NCI}-\mathrm{H} 460\left(\operatorname{lgGI}_{50}=-5.58 ;\right.$ $\left.\operatorname{lgTGI}=-4.93 ; \operatorname{lgLC}_{50}=-4.37\right)$, NCI-H522 $\left(\operatorname{lgGI}_{50}=-5.84\right.$; $\operatorname{lgTGI}=-5.08 ; \operatorname{lgLC} \mathrm{C}_{50}=-4.31$; COLO 205 colon cancer $\left(\operatorname{lgGI}_{50}=-5.89 ; \operatorname{lgTGI}=-5.14 ; \operatorname{lgLC} C_{50}=-4.36\right)$, HCC-2998 $\left(\operatorname{lgGI}_{50}=-5.78 ; \operatorname{lgTGI}=-4.95 ; \operatorname{lgL} \mathrm{C}_{50}=-4.36\right)$, 
HCT-116 $\left(\operatorname{lgGI}_{50}=-6.04 ; \lg \mathrm{TGI}=-4.90 ; \operatorname{lgLC} \mathrm{C}_{50}=-4.42\right)$, HT29 $\left(\operatorname{lgGI}{ }_{50}=-6.29 ; \operatorname{lgTGI}=-4.97 ; \operatorname{lgLC}_{50}=-4.31\right)$, $\mathrm{KM} 12\left(\operatorname{lgGI}_{50}=-6.02 ; \operatorname{lgTGI}=-4.77 ; \operatorname{lgLC}_{50}=-4.31\right)$; SF-268 CNS cancer $\left(\operatorname{lgGI}{ }_{50}=-5.26\right.$; $\lg \mathrm{TGI}=-4.58$; $\left.\operatorname{lgLC} \mathrm{C}_{50}=-4.08\right), \mathrm{SF}-295\left(\operatorname{lgGI} \mathrm{I}_{50}=-6.07 ; \operatorname{lgTGI}=-4.98\right.$; $\left.\operatorname{lgLC}_{50}=-4.43\right), \mathrm{SF}-539\left(\operatorname{lgGI} \mathrm{I}_{50}=-5.83 ; \operatorname{lgTGI}=-5.25\right.$; $\left.\operatorname{lgLC}_{50}=-4.57\right), \mathrm{SNB}-19\left(\operatorname{lgGI} \mathrm{I}_{50}=-5.65 ; \operatorname{lgTGI}=-4.62\right.$; $\left.\operatorname{lgLC}_{50}=-4.06\right)$, SNB-75 $\left(\operatorname{lgGI} \mathrm{I}_{50}=-5.74 ; \lg \mathrm{TGI}=-5.09 ;\right.$ $\left.\operatorname{lgLC}_{50}=-4.41\right), \mathrm{U} 251\left(\operatorname{lgGI}{ }_{50}=-6.01 ; \lg \mathrm{TGI}=-4.80\right.$ $\left.\operatorname{lgLC}_{50}=-4.34\right)$; LOX IMVI melanoma $\left(\operatorname{lgGI}_{50}=-5.81\right.$; $\left.\operatorname{lgTGI}=-4.74 ; \operatorname{lgLC}_{50}=-4.28\right)$, MALME-3M $\left(\operatorname{lgGI}_{50}=-4.93\right.$; $\left.\operatorname{lgTGI}=-4.52 ; \operatorname{lgLC} C_{50}=-4.10\right), \mathrm{M} 14\left(\operatorname{lgGI}_{50}=-6.24\right.$; $\left.\operatorname{lgTGI}=-4.84 ; \operatorname{lgLC}_{50}=-4.36\right), \mathrm{MDA}^{\mathrm{MB}}-435\left(\operatorname{lgGI}_{50}=\right.$ $\left.-6.64 ; \lg \mathrm{TGI}=-6.09 ; \operatorname{lgLC}_{50}=-4.47\right)$, SK-MEL-2 $\left(\operatorname{lgGI}_{50}=\right.$ -6.05; $\left.\operatorname{lgTGI}=-4.72 ; \operatorname{lgLC}_{50}=-4.27\right)$, SK-MEL-28 $\left(\operatorname{lgGI}_{50}=\right.$ -5.29; $\left.\operatorname{lgTGI}=-4.66 ; \operatorname{lgLC}_{50}=-4.27\right)$, SK-MEL-5 $\left(\operatorname{lgGI}_{50}=\right.$ -5.74; $\left.\operatorname{lgTGI}=-4.94 ; \operatorname{lgLC}_{50}=-4.47\right)$, UACC-257 $\left(\operatorname{lgGI}_{50}=\right.$ -4.84; $\left.\operatorname{lgTGI}=-4.47 ; \operatorname{lgLC}_{50}=-4.09\right)$, UACC-62 $\left(\operatorname{lgGI}_{50}=\right.$ -6.07; lgTGI $\left.=-4.78 ; \operatorname{lgLC}_{50}=-4.26\right)$, 786-0 renal cancer $\left(\operatorname{lgGI} \mathrm{I}_{50}=-5.29 ; \operatorname{lgTGI}=-4.69 ; \operatorname{lgLC}_{50}=-4.30\right)$, A498 $\left(\operatorname{lgGI}_{50}=-6.38 ; \lg \mathrm{TGI}=-5.22 ; \operatorname{lgLC}_{50}=-4.46\right)$, RFX $393\left(\operatorname{lgGI}_{50}=-5.84 ; \lg \mathrm{TGI}=-5.25 ; \operatorname{lgLC}_{50}=-4.57\right)$, $\mathrm{SN} 12 \mathrm{C}\left(\operatorname{lgGI}_{50}=-5.57 ; \lg \mathrm{TGI}=-4.69 ; \operatorname{lgLC}_{50}=-4.27\right)$, UO-31 $\left(\operatorname{lgG}_{\mathrm{I} 50}=-5.38 ; \lg \mathrm{TGI}=-4.66 ; \operatorname{lgLC}_{50}=-4.20\right)$, MCF7 breast cancer $\left(\operatorname{lgGI} \mathrm{I}_{50}=-6.17\right.$; $\operatorname{lgTGI}=-4.74$; $\left.\operatorname{lgLC}_{50}=-4.24\right)$, MDA-MB-231/ATCC $\left(\operatorname{lgGI}{ }_{50}=-6.13\right.$; $\left.\operatorname{lgTGI}=-4.82 ; \operatorname{lgLC}_{50}=-4.27\right), \mathrm{BT}-549\left(\operatorname{lgGI}_{50}=-5.22\right.$; $\left.\operatorname{lgTGI}=-4.66 ; \operatorname{lgLC}_{50}=-4.31\right)$, MDA-MB-468 $\left(\operatorname{lgGI}_{50}=\right.$ -6.47; lgTGI = -5.40; $\operatorname{lgLC}_{50}=-4.15$ ).

Compound $\mathbf{3 g}$ had the anticarcinogenic activity against MDA-MB-435 melanoma $\left(\operatorname{lgGI}_{50}=-6.44\right.$; $\lg \mathrm{TGI}=$ -5.77; $\operatorname{lgLC}_{50}=-5.00$ ).

Thus, derivatives of 3-R-6-(4-methoxyphenyl)$7 H$-[1,2,4] triazolo[3,4- $b][1,3,4]$ thiadiazine exhibit the antineoplastic activity against a wide range of cancer cells lines and can become the basis for creating new effective anticancer agents.

\section{Experimental part}

\section{Chemistry part}

4-Amino-5-R-4H-1,2,4-triazole-3-thiols 2b-g were synthesized according to the method described in [18], 4-amino-4H-1,2,4-triazole-3-thiol (2a) was synthesized by the method [19].

${ }^{1} \mathrm{H}$ NMR spectra were recorded on a Bruker VXR300 (Germany), the working frequency $-299.945 \mathrm{MHz}$, in DMSO- $d_{6}$ using TMS as an internal standard. Chemical shifts were reported in ppm units using the $\delta$ scale.

The melting points were measured on a smallsized heating table with a RNMK 05 observation device (VEB Analytik, Dresden).

The general procedure for the synthesis of 3-R-6-(4-methoxyphenyl)-7H-[1,2,4]triazolo[3,4- $b]$ $[1,3,4]$ thiadiazines $3 \mathbf{a}-\mathbf{g}$. The mixture of the appropriate 4-amino-4H-1,2,4-triazole-3-thiol $2 \mathbf{a}-\mathbf{g}(0.01 \mathrm{~mol})$ and 4-methoxyphenacyl bromide (2.29 g; $0.01 \mathrm{~mol}$ ) was refluxed in $50 \mathrm{~mL}$ of ethyl acetate during 2 hours. After cooling the precipitate of salts $\mathbf{3 a - g}$ was filtered off, washed with ethyl acetate, dried on air and recrystallized from ethanol.

6-(4-Methoxyphenyl)-7H-[1,2,4]triazolo[3,4-b] $[1,3,4]$ thiadiazine hydrobromide (3a). Yield - $2.59 \mathrm{~g}$ (79\%). M. p. $142-144^{\circ} \mathrm{C}$ (from ethanol). Anal. Calcd. for $\mathrm{C}_{11} \mathrm{H}_{11} \mathrm{BrN}_{4} \mathrm{OS}, \%$ : N 17.12. Found, \%: N 17.01. ${ }^{1} \mathrm{H}$ NMR (300 MHz, DMSO- $d_{6}$ ), $\delta$, ppm: $3.86\left(3 \mathrm{H}, \mathrm{s}, \mathrm{OCH}_{3}\right) ; 4.41$ $\left(2 \mathrm{H}, \mathrm{s}, \mathrm{SCH}_{2}\right) ; 7.12$ and $7.97\left(2 \mathrm{H}, \mathrm{d}, J=8.7 \mathrm{~Hz}, \mathrm{C}_{6} \mathrm{H}_{4}\right)$; $9.12(1 \mathrm{H}, \mathrm{s}, 3-\mathrm{CH})$.

6-(4-Methoxyphenyl)-3-methyl-7H-[1,2,4]triazolo[3,4-b][1,3,4]thiadiazine hydrobromide (3b). Yield - 2.76 g (81\%). M. p. $205-207^{\circ} \mathrm{C}$ (from ethanol). Anal. Calcd. for $\mathrm{C}_{12} \mathrm{H}_{13} \mathrm{BrN}_{4} \mathrm{OS}, \%$ : N 16.42. Found, \%: N 16.60. ${ }^{1} \mathrm{H}$ NMR (300 MHz, DMSO- $d_{6}$ ), $\delta$, ppm: 2.60 $\left(3 \mathrm{H}, \mathrm{s}, \mathrm{CH}_{3}\right) ; 3.87\left(3 \mathrm{H}, \mathrm{s}, \mathrm{OCH}_{3}\right) ; 4.45\left(2 \mathrm{H}, \mathrm{s}, \mathrm{SCH}_{2}\right)$; 7.14 and $8.05\left(2 \mathrm{H}, \mathrm{d}, J=8.7 \mathrm{~Hz}, \mathrm{C}_{6} \mathrm{H}_{4}\right)$.

3-Ethyl-6-(4-methoxyphenyl)-7H-[1,2,4]triazolo[3,4-b][1,3,4]thiadiazine hydrobromide (3c). Yield - $2.94 \mathrm{~g}(83 \%)$. M. p. $236-238^{\circ} \mathrm{C}$ (from ethanol). Anal. Calcd. for $\mathrm{C}_{13} \mathrm{H}_{15} \mathrm{BrN}_{4} \mathrm{OS}, \%$ : N 15.77. Found, \%: N 15.72. ${ }^{1} \mathrm{H}$ NMR (300 MHz, DMSO- $d_{6}$ ), $\delta$, ppm: 1.33 $\left(3 \mathrm{H}, \mathrm{t}, J=7.6 \mathrm{~Hz}, \mathrm{CH}_{3}\right) ; 3.00\left(2 \mathrm{H}, \mathrm{q}, J=7.6 \mathrm{~Hz}, \mathrm{CH}_{2}\right)$; $3.87\left(3 \mathrm{H}, \mathrm{s}, \mathrm{OCH}_{3}\right) ; 4.46\left(2 \mathrm{H}, \mathrm{s}, \mathrm{SCH}_{2}\right) ; 7.15$ and 8.04 $\left(2 \mathrm{H}, \mathrm{d}, J=9.0 \mathrm{~Hz}, \mathrm{C}_{6} \mathrm{H}_{4}\right)$.

6-(4-Methoxyphenyl)-3-propyl-7H-[1,2,4]triazolo[3,4-b][1,3,4]thiadiazine hydrobromide (3d). Yield - $2.91 \mathrm{~g}(79 \%)$. M. p. $212-214^{\circ} \mathrm{C}$ (from ethanol). Anal. Calcd. for $\mathrm{C}_{14} \mathrm{H}_{17} \mathrm{BrN}_{4} \mathrm{OS}, \%$ : N 15.17. Found, \%: N 15.10. ${ }^{1} \mathrm{H}$ NMR (300 MHz, DMSO- $d_{6}$ ), $\delta$, ppm: 1.06 $\left(3 \mathrm{H}, \mathrm{t}, J=7.6 \mathrm{~Hz}, \mathrm{CH}_{3}\right) ; 1.81-1.91\left(2 \mathrm{H}, \mathrm{m}, \mathrm{CH}_{2}\right) ; 3.01$ $\left(2 \mathrm{H}, \mathrm{t}, J=8.0 \mathrm{~Hz}, \mathrm{CH}_{2}\right) ; 3.87\left(3 \mathrm{H}, \mathrm{s}, \mathrm{OCH}_{3}\right) ; 4.46(2 \mathrm{H}, \mathrm{s}$, $\left.\mathrm{SCH}_{2}\right) ; 7.06$ and $8.04\left(2 \mathrm{H}, \mathrm{d}, \mathrm{J}=8.8 \mathrm{~Hz}, \mathrm{C}_{6} \mathrm{H}_{4}\right)$.

6-(4-Methoxyphenyl)-3-butyl-7H-[1,2,4]triazolo[3,4-b][1,3,4]thiadiazine hydrobromide (3e). Yield - $2.99 \mathrm{~g}(78 \%)$. M. p. $193-195^{\circ} \mathrm{C}$ (from ethanol). Anal. Calcd. for $\mathrm{C}_{15} \mathrm{H}_{19} \mathrm{BrN}_{4} \mathrm{OS}, \%$ : N 14.62. Found, \%: $\mathrm{N}$ 14.83. ${ }^{1} \mathrm{H}$ NMR (300 MHz, DMSO- $d_{6}$ ), $\delta$, ppm: 0.94 $\left(3 \mathrm{H}, \mathrm{t}, J=7.6 \mathrm{~Hz}, \mathrm{CH}_{3}\right) ; 1.35-1.44\left(2 \mathrm{H}, \mathrm{m}, \mathrm{CH}_{2}\right) ; 1.69-1.77$ $\left(2 \mathrm{H}, \mathrm{m}, \mathrm{CH}_{2}\right) ; 2.92\left(2 \mathrm{H}, \mathrm{t}, J=8.0 \mathrm{~Hz}, \mathrm{CH}_{2}\right) ; 3.86(3 \mathrm{H}$, s, $\left.\mathrm{OCH}_{3}\right) ; 4.36\left(2 \mathrm{H}, \mathrm{s}, \mathrm{SCH}_{2}\right) ; 7.08$ and $7.92(2 \mathrm{H}, \mathrm{d}$, $\left.J=8.8 \mathrm{~Hz}, \mathrm{C}_{6} \mathrm{H}_{4}\right)$.

6-(4-Methoxyphenyl)-3-pentyl-7H-[1,2,4]triazolo[3,4-b][1,3,4]thiadiazine hydrobromide (3f). Yield - $2.70 \mathrm{~g}(68 \%)$. M. p. $185-187^{\circ} \mathrm{C}$ (from ethanol). Anal. Calcd. for $\mathrm{C}_{16} \mathrm{H}_{21} \mathrm{BrN}_{4} \mathrm{OS}, \%$ : N 14.10. Found, \%: N 14.03. ${ }^{1} \mathrm{H}$ NMR (300 MHz, DMSO- $d_{6}$ ), $\delta$, ppm: 0.94 $\left(3 \mathrm{H}, \mathrm{t}, J=7.6 \mathrm{~Hz}, \mathrm{CH}_{3}\right) ; 1.34-1.45\left(4 \mathrm{H}, \mathrm{m}, 2 \times \mathrm{CH}_{2}\right)$; 1.75-1.83 (2H, m, $\left.\mathrm{CH}_{2}\right) ; 2.94\left(2 \mathrm{H}, \mathrm{t}, \mathrm{J}=8.0 \mathrm{~Hz}, \mathrm{CH}_{2}\right)$; $3.89\left(3 \mathrm{H}, \mathrm{s}, \mathrm{OCH}_{3}\right) ; 4.32\left(2 \mathrm{H}, \mathrm{s}, \mathrm{SCH}_{2}\right) ; 7.03$ and 7.98 $\left(2 \mathrm{H}, \mathrm{d}, J=8.8 \mathrm{~Hz}, \mathrm{C}_{6} \mathrm{H}_{4}\right)$.

6-(4-Methoxyphenyl)-3-(trifluoromethyl)-7H$[1,2,4]$ triazolo[3,4-b] $[1,3,4]$ thiadiazine hydrobromide $(3 g)$. 
Yield - 2.84 g (72\%). M. p. $162-164^{\circ} \mathrm{C}$ (from ethanol). Anal. Calcd. for $\mathrm{C}_{12} \mathrm{H}_{10} \mathrm{BrF}_{3} \mathrm{~N}_{4} \mathrm{OS}$, \%: $\mathrm{N}$ 14.18. Found, \%: N 14.45. ${ }^{1} \mathrm{H}$ NMR (300 MHz, DMSO- $d$ ), $\delta$, ppm: $3.88\left(3 \mathrm{H}, \mathrm{s}, \mathrm{OCH}_{3}\right) ; 4.48\left(2 \mathrm{H}, \mathrm{s}, \mathrm{SCH}_{2}\right) ; 7.11$ and 7.98 $\left(2 \mathrm{H}, \mathrm{d}, J=9.0 \mathrm{~Hz}, \mathrm{C}_{6} \mathrm{H}_{4}\right)$.

\section{Biology Part}

The methodology of the NCI procedure for the primary anticancer assay is detailed in Anticancer Drug Development Guide [14]. Briefly, the protocol was performed on 60 human tumor cell lines derived from different nine neoplastic diseases. NCI-60 testing was performed as a single concentration, which was tested on all 60 cell lines in a single dose of $10^{-5} \mathrm{M}$. All of the assays were in accordance with the protocol of the Drug Evaluation Branch, National Cancer Institute, Bethesda, USA. If the results obtained met selection criteria, then the compound was tested again on all 60 cell lines in $5 \times 10$-fold dilutions with the top dose being $10^{-4} \mathrm{M}$.

\section{Conclusions}

1. A series of 3-R-6-(4-methoxyphenyl)-7H-[1,2,4]triazolo[3,4- $b][1,3,4]$ thiadiazines has been synthesized by the interaction of 4-amino-5-R-4H-1,2,4-triazole3 -thiols with 4-methoxyphenacyl bromide in ethyl acetate. The structure and purity of all the products have been confirmed by ${ }^{1} \mathrm{H}$ NMR spectroscopy and elemental analysis.

2. Derivatives of 3-R-6-(4-methoxyphenyl)-7H$[1,2,4]$ triazolo[3,4- $b][1,3,4]$ thiadiazine have been proven to have the high level of the antitumor activity against leukemia, non-small cell lung cancer, colon cancer, CNS cancer, melanoma, ovarian cancer, renal cancer, prostate cancer and breast cancer, and thus, can be recommended for in-depth preclinical studies.

Conflict of interests: the authors have no conflict of interests to declare.

\section{References}

1. Cancer. https://www.who.int/news-room/fact-sheets/detail/cancer (accessed Apr 10, 2020).

2. Plummer, M.; de Martel, C.; Vignat, J.; Ferlay, J.; Bray, F.; Franceschi, S. Global burden of cancers attributable to infections in 2012: a synthetic analysis. Lancet Glob. Health 2016, 4 (9), e609-e616. https://doi.org/10.1016/S2214-109X(16)30143-7.

3. Ogasa, Y.; Kuwamura, T.; Akiyoshi, T.; Murakami, S.; Tanaka, H.; Umeda, S.; Suematsu F. Evaluation of patient adherence to anastrozole therapy for breast cancer. Gan To Kagaku Ryoho 2018, 45 (6), 965-968.

4. Barros-Oliveira, M. d. C.; Costa-Silva, D. R.; Andrade, D. B. d.; Borges, U. S.; Tavares, C. B.; Borges, R. S.; Silva, J. d. M.; Silva, B. B. d. Use of anastrozole in the chemoprevention and treatment of breast cancer: A literature review. Revista da Associação Médica Brasileira 2017, 63, 371-378. http:// dx.doi.org/10.1590/1806-9282.63.04.371.

5. The ATAC (Arimidex, Tamoxifen Alone or in Combination) Trialists' Group. Anastrozole alone or in combination with tamoxifen versus tamoxifen alone for adjuvant treatment of postmenopausal women with early-stage breast cancer. Cancer 2003, 98 (9), 1802-1810. https://doi.org/10.1002/ cncr.11745.

6. Kravchenko, T. V.; Panasenko, O. I.; Knysh, E. G. Biological activity of the derivatives of 1,2,4-triazole. Farmatsevtychnyi zhurnal 2018, 5, 25-30. https://doi.org/10.32352/0367-3057.5.16.02.

7. The BIG 1-98 Collaborative Group. Letrozole Therapy Alone or in Sequence with Tamoxifen in Women with Breast Cancer. N. Engl. J. Med. 2009, 361 (8), 766-776. https://doi.org/10.1056/NEJMoa0810818.

8. Aggarwal, N.; Kumar, R.; Dureja, P.; Khurana, J. M. Synthesis, antimicrobial evaluation and QSAR analysis of novel nalidixic acid based 1,2,4-triazole derivatives. Eur. J. Med. Chem. 2011, 46 (9), 4089-4099. https://doi.org/10.1016/j.ejmech.2011.06.009.

9. Kumar, D.; Narayanam, M. K.; Chang, K.-H.; Shah, K. Synthesis of Novel Indolyl-1,2,4-triazoles as Potent and Selective Anticancer Agents. Chemical Biology \& Drug Design 2011, 77 (3), 182-188. https://doi.org/10.1111/j.1747-0285.2010.01051.x.

10. Khan, I.; Zaib, S.; Ibrar, A.; Rama, N. H.; Simpson, J.; Iqbal, J. Synthesis, crystal structure and biological evaluation of some novel 1,2,4-triazolo[3,4-b]1,3,4-thiadiazoles and 1,2,4-triazolo[3,4-b]-1,3,4-thiadiazines. Eur. J. Med. Chem. 2014, 78, 167-177. https://doi.org/10.1016/j.ejmech.2014.03.046.

11. Purohit, D. H.; Dodiya, B. L.; Ghetiya, R. M.; Vekariya, P. B.; Joshi H. S. Synthesis and antimicrobial activity of some new 1,3,4-thiadiazoles and 1,3,4-thiadiazines containing 1,2,4-triazolo nucleus. Acta Chim. Slov. 2011, 58, 53-59.

12. Prakash, O.; Aneja, D. K.; Hussain, K.; Lohan, P.; Ranjan, P.; Arora, S.; Sharma, C.; Aneja, K. R. Synthesis and biological evaluation of dihydroindeno and indeno[1,2-e][1,2,4]triazolo[3,4-b][1,3,4]thiadiazines as antimicrobial agents. Eur. J. Med. Chem. 2011, 46 (10), 5065-5073. https://doi. org/10.1016/j.ejmech.2011.08.019.

13. El Shehry, M. F.; Abu-Hashem, A. A.; El-Telbani, E. M. Synthesis of 3-((2,4-dichlorophenoxy)methyl)-1,2,4-triazolo(thiadiazoles and thiadiazines) as anti-inflammatory and molluscicidal agents. Eur. J. Med. Chem. 2010, 45 (5), 1906-1911. https://doi.org/10.1016/j.ejmech.2010.01.030.

14. Teicher, B. A.; Andrews, P. A., Eds. Anticancer Drug Development Guide; Humana Press: 2004.

15. Alley, M. C.; Scudiero, D. A.; Monks, A.; Hursey, M. L.; Czerwinski, M. J.; Fine, D. L.; Abbott, B. J.; Mayo, J. G.; Shoemaker, R. H.; Boyd, M. R. Feasibility of Drug Screening with Panels of Human Tumor Cell Lines Using a Microculture Tetrazolium Assay. Cancer Res. 1988,48 (3), $589-601$.

16. Carter, P. H.; Scherle, P. A.; Muckelbauer, J. A.; Voss, M. E.; Liu, R.-Q.; Thompson, L. A.; Tebben, A. J.; Solomon, K. A.; Lo, Y. C.; Li, Z.; Strzemienski, P.; Yang, G.; Falahatpisheh, N.; Xu, M.; Wu, Z.; Farrow, N. A.; Ramnarayan, K.; Wang, J.; Rideout, D.; Yalamoori, V.; Domaille, P.; Underwood, D. J.; Trzaskos, J. M.; Friedman, S. M.; Newton, R. C.; Decicco, C. P. Photochemically enhanced binding of small molecules to the tumor necrosis factor receptor-1 inhibits the binding of TNF- $\alpha$. Proc. Natl. Acad. Sci. U.S.A. 2001, 98 (21), 11879-11884. https://doi.org/10.1073/pnas.211178398.

17. Grever, M. R.; Schepartz, S. A.; Chabner, B. A. The National Cancer Institute: cancer drug discovery and development program. Semin. Oncol. 1992, $19(6), 622-638$

18. Hou, N.; Xu, L. J. Synthesis and bacteriostatic activity of new thiosemicarbazone derivatives - aminomercaptotriazole Schiff bases. Yaoxue Xuebao 1992, 27 (10), 738-742.

19. 5-( $\beta$-hydroxyethylthio)-4-amino-1,2,4-(4H)-triazol. In Sintezy geterotsiklicheskikh soyedineniy; Mndzhoyan, A. L., Ed.; Academy of Sciences of Armenian SSR: Yerevan, 1964; Vol. 6, pp. 41-43.

The work was carried out according to the research plan of the Nizhyn Mykola Gogol State University on the topic "Synthesis of novel sulphur and nitrogen containing heterocyclic compounds and investigation of their practically useful properties" (the state registration No. 0115U005451; the research period: 2015-2019). 